\title{
EXPERIENCIAS DIGITALES, RIESGOS Y ENFOQUE EDUCATIVO DEL OCIO DIGITAL CON JÓVENES EN DIFICULTAD SOCIAL
}

\author{
DIGITAL EXPERIENCES, RISKS AND EDUCATIONAL APPROACH TO DIGITAL \\ LEISURE WITH YOUNG PEOPLE IN SOCIAL DIFFICULTY
}

\section{EXPERIÊNCIAS DIGITAIS, RISCOS E ABORDAGEM EDUCACIONAL PARA O LAZER DIGITAL COM JOVENS EM DIFICULDADE SOCIAL}

\author{
Margarita Vasco-González \\ Universidad Nacional de Educación a Distancia, Espanha \\ margavasco@gmail.com \\ Rosa Goig Martínez \\ Universidad Nacional de Educación a Distancia, Espanha \\ rmgoig@uned.es \\ Álvaro Manuel Úbeda-Sánchez \\ Universidad de Granada, Espanha \\ amsu@correo.ugr.es
}

RESUMEN: Las tecnologías de la información y comunicación pueden ser una herramienta para el desarrollo de valores sociales y educativos que faciliten a los jóvenes en dificultad social las oportunidades necesarias para su inclusión en la sociedad contemporánea. Desde esta perspectiva, se plantea un trabajo cuyo objetivo es investigar las experiencias de ocio digital de los jóvenes en dificultad social para descubrir las oportunidades educativas que se pueden implementar en el colectivo a través de actividades de ocio sostenidas en el entorno tecnológico. Es un estudio cualitativo que utiliza como instrumentos de recogida de datos las entrevistas abiertas realizadas a 78 informantes. Entre los resultados relevantes obtenidos destaca que este colectivo de jóvenes de exclusión social permanece en conexión constante con los amigos a través de su Smartphone. Mediante el uso continuado de este dispositivo alternan sus ocupaciones laborales, familiares y formativas con las redes sociales e internet para buscar, entre otras acciones, información sobre famosos o "YouTubers" de moda para copiar su comportamiento y estilo personal. Por ello, se concluye con el análisis sobre un enfoque educativo que utilice estos dispositivos como medio para trabajar, con los jóvenes en dificultad social, alternativas educativas de ocio digital que les faciliten oportunidades para el desarrollo de actuaciones en la red, cuyo valor para el crecimiento personal y social posibilite nuevas oportunidades para la inclusión en la sociedad.

PALABRAS CLAVE: Ocio digital. Jóvenes en dificultad social. Práctica educativa. Inclusión. Enfoque educativo.

ABSTRACT: Information and communication technologies can be a tool for the development of social and educational values that provide young people with social difficulties with the necessary opportunities for their insertion in contemporary society. In this perspective, a work is proposed whose objective is to investigate the digital leisure experiences of young people in social difficulties to discover the educational opportunities 
that can be implemented in the group through leisure activities sustained in the technological environment. This is a qualitative study, with open data interviews conducted with 78 informants as data collection instruments. Among the relevant results obtained is that this group of young people in a situation of social exclusion remains in constant contact with friends through their smartphones. With the continued use of this device, they alternate their professional, family and educational occupations with social networks and the Internet to search, among other actions, information about celebrities or fashion "youtubers" to copy their personal behavior and style. Therefore, we conclude with the analysis of an educational approach that uses these devices as a means of working with young people in social difficulty, educational alternatives for digital leisure that provide opportunities for the development of actions on the network, whose value for personal and social growth enables new opportunities for inclusion in society.

KEYWORDS: Digital Leisure. Young People in Social Difficulty. Educative Practice. Inclusion. Educational approach.

RESUMO: As tecnologias de informação e comunicação podem ser uma ferramenta para o desenvolvimento de valores sociais e educacionais que proporcionem aos jovens com dificuldades sociais as oportunidades necessárias para a sua inserção na sociedade contemporânea. Nessa perspectiva, é proposto um trabalho cujo objetivo é investigar as experiências de lazer digital de jovens em dificuldades sociais para descobrir as oportunidades educacionais que podem ser implementadas no grupo por meio de atividades de lazer sustentadas no ambiente tecnológico. Trata-se de um estudo qualitativo, tendo como instrumentos de coleta de dados entrevistas abertas realizadas com 78 informantes. Entre os resultados relevantes obtidos está que este grupo de jovens em situação de exclusão social se mantém em constante ligação com os amigos através do seu smartphone. Com o uso contínuo desse dispositivo, eles alternam suas ocupações profissional, familiar e educacional com as redes sociais e a Internet para buscar, entre outras ações, informações sobre celebridades ou "youtubers" da moda para copiar seu comportamento e estilo pessoal. Portanto, concluímos com a análise de uma abordagem educacional que utiliza esses dispositivos como meio de trabalhar com jovens em dificuldade social, alternativas educacionais para o lazer digital que proporcionam oportunidades para o desenvolvimento de ações na rede, cujo valor para o crescimento pessoais e sociais possibilitam novas oportunidades de inclusão na sociedade.

PALAVRAS-CHAVE: Lazer digital. Jovens em dificuldade social. Prática educacional. Inclusão. Abordagem educacional.

\section{Introducción y revisión de la literatura}

El cambio producido en las prácticas de ocio en los jóvenes se hace visible en el entorno tecnológico por las múltiples opciones lúdicas que surgen a diario dentro de la red. Estas experiencias virtuales concentran, en la actualidad, distintas investigaciones que se abordan desde perspectivas diferenciadas (CUBEROS et al., 2018; RODRÍGUEZ; BALLESTEROS, 2019; PONCE-DE-LEÓN-ELIZONDO et al., 2019; VASCO-GONZÁLEZ, GOIG; ÁLVAREZ, 2020). Cabe señalar el reconocimiento de un colectivo de jóvenes que también pertenece a esta generación hiperconectada pero que, dadas sus características de dificultad social entendida como un déficit en sus experiencias familiares: violencia en 
el hogar, desestructuración familiar; laborales: desempleo o precariedad; sociales: criminalidad, marginalidad y educativas: falta de formación absentismo, analfabetismo... tienen grandes dificultades para aprovechar de forma competente las oportunidades que les brinda el entorno social y tecnológico en el que participan y buscan en el ocio digital recursos fáciles y de escasa trascendencia para escapar de la complejidad de su entorno (GONZÁLEZ, 2013). Estas formas de practicar el ocio tienen implicaciones en sus procesos de socialización ya que limitan las oportunidades vinculadas al ocio por Cuenca (2014, p. 26) para "el desarrollo de valores positivos para la persona, así como para la sociedad en la que participa".

Atendiendo a esta situación se observa la posibilidad de contemplar estas prácticas como ámbito pedagógico para el fortalecimiento de esas oportunidades mediante el desarrollo de los valores sociales, educativos y ciudadanos que describen Tumino y Bournissen (2017, p. 195) asociados al ocio digital, repercutiendo con ello en la mejora de la realidad de este colectivo en todos los ámbitos de su vida.

\subsection{Prácticas de ocio digital en los jóvenes en dificultad social}

Para hacer realidad la experiencia educativa del ocio digital entre los jóvenes en dificultad social es necesario partir del estudio de las formas de uso que hacen estos jóvenes de este espacio virtual y las posibles repercusiones de las mismas en su desarrollo personal y social. En este sentido, se comprueba que los patrones de uso de las tecnologías digitales ${ }^{1}$ al servicio del ocio son muy similares al resto de población juvenil (MELENDRO; GARCÍA-CASTILLA; GOIG, 2016) pero manifiestan menores competencias para hacer de ese ocio una experiencia con valor para su crecimiento personal y social (VASCO-GONZÁLEZ, 2018). Así, como señalan los autores citados, el uso del móvil con conexión a internet o Smartphone y de las redes sociales virtuales se confirma como el soporte esencial para las prácticas de ocio digital dentro de este colectivo y, como defiende Vázquez (2019, p. 87) su uso tiene principalmente una función comunicativa vinculada a las actividades de entretenimiento.

Por otro lado, como recogen Pastor, Martín y Montes (2019, p. 1006) los jóvenes muestran una tendencia general por la necesidad de llevar siempre el móvil para no perderse nada de lo que sucede en la red, a la que relacionan con la posibilidad de quedarse fuera del grupo. De esta forma operan con su Smartphone en el entorno tecnológico manifestando, en algunos casos, las posibles implicaciones derivadas de este comportamiento asociadas a uno de los riesgos que más se vincula al contexto online, la dependencia (CABERO et al., 2020). Entre las consecuencias que se reconocen procedentes del uso continuado y adictivo a la red se señala: baja autoestima y aislamiento social, familiar y escolar que conlleva la pérdida de los lazos afectivos y la aparición de problemas sociales (HERNÁNDEZ et al., 2019) haciendo de los jóvenes en dificultad social, un colectivo más vulnerable aún si cabe a las posibles incidencias de estas prácticas digitales.

Otra cuestión que preocupa en la literatura analizada, es la que recoge la inquietud sobre las posibilidades que se dan en las redes sociales e internet para difundir mentiras e información falsa (CARO, 2015; CALVO, LUQUE, 2019) y de las dificultades que manifiestan, principalmente los jóvenes, para reconocer este tipo de informaciones, ya

\footnotetext{
1 Todo soporte digital con conexión a internet.
} 
que requieren el desarrollo de la competencia digital (AVILÉS, 2018), elemento que no se reconoce entre los jóvenes en dificultad pese a mostrar un "dominio natural tecnológico de las herramientas digitales" (GRANADO, 2019, p. 32).

Continuando en esa línea se alude a la cantidad de información y contenidos que circulan por el entorno virtual, sometiendo a los jóvenes en sus prácticas digitales de ocio a una saturación de datos y mensajería que Area y Guarro (2012, p. 48) denominan "infoxicación" que desencadenan, como asegura Alarcón (2019, p. 180) procesos de difusión y producción de información que solamente generan confusión e ignorancia. En este sentido, este exceso de información ocasiona, además, una actitud de pasividad que fomenta que los jóvenes no comprueben la veracidad de la misma limitándose a reenviar, a modo de entretenimiento, la noticia o contenido recibido alimentando, de esta manera, lo que se conoce como desinformación (SANMARTíN; MEGÍAS, 2020).

Por otro lado, el peligro de esta trasmisión incontrolada de noticias falsas ha favorecido como señala (CARO, 2015, p. 189) la propagación de lo que se conoce como bulo, en referencia a las noticas o publicaciones con contenidos de dudosa procedencia, y los rumores, siendo los jóvenes los que menores capacidades muestran para distinguirlos de forma crítica (BALLESTEROS; PICAZO, 2018). Las repercusiones de esta difusión indiscriminada de falsas historias entre los jóvenes pueden desencadenar, como alerta el estudio de Rivadulla López y Rodríguez Correa (2019, p. 181), en un proceso de aislamiento social que pone en serio riesgo la integridad de muchos jóvenes que carecen de entornos de protección como es el caso de los jóvenes en dificultad social. A ello se suma también el estudio de González y Campoy (2018) que señala la difusión de bromas, rumores, secretos y cotilleos por parte de los jóvenes a través de la red como conductas que pueden llegar a derivar en procesos de ciberacoso.

Si bien es cierto que las evidencias científicas aluden a los posibles riesgos de las prácticas digitales de los jóvenes, también se encuentran trabajos que sugieren la posibilidad de contrarrestar estos riesgos mediante un buen uso de las redes sociales y tecnologías, como el realizado por Prats et al. (2018) a través de su propuesta de talleres educativos para formar y sensibilizar a alumnos de educación secundaria y bachillerato sobre el uso adecuado y seguro de las redes sociales que obtuvo valoraciones muy positivas. Es aquí donde tiene cabida nuestra propuesta ya que como hemos observado apenas existen aportaciones de corte científico que acojan propuestas para el trabajo socioeducativo en el ocio digital con jóvenes en dificultad social.

\subsection{Practicas socioeducativas para el ocio digital en los jóvenes en dificultad social}

Atendiendo a lo expuesto en líneas anteriores y de acuerdo con De Valenzuela Bandín, Gradaílle Pernas y Caride Gómez (2018, p. 43) el trabajo socioeducativo con jóvenes en dificultad social orientado a las prácticas de ocio suele centrarse en llenar el vacío temporal más que en aportar una visión educativa. Es por ello que Maroñas Bermúdez, Martínez García, y Gradaílle Pernas (2019, p. 118) ponen de manifiesto la importancia de educar desde la pedagogía del ocio mediante propuestas socioeducativas que permitan, a los jóvenes en dificultad, ocupar estos espacios con experiencias que les ayuden en su crecimiento personal para el mañana ya que, como afirman las autoras, la educación en el ocio es un espacio privilegiado para la promoción de experiencias con 
fines educativos, culturales y sociales por ser el más cercano y cotidiano a la persona. Desde esta perspectiva la educación en el ocio debe tener en cuenta que para los jóvenes uno de esos espacios cotidianos se ubica actualmente entre las tecnologías de información y comunicación y las redes sociales que Rodríguez y Ballesteros (2019, p. 20) observan también vinculadas al ocio como medio para ayudar a ampliar oportunidades.

Como se ha comprobado son numerosas las publicaciones que se centran en el valor de las tecnologías y redes sociales para trabajar en contextos educativos formales cercanos a la educación superior (BERTONE et al., 2019; MARÍN-DIAZ; CABEROALMENARA, 2019; PEÑA RUEDA; PEGALAJAR, 2018; SÁNCHEZ PADILLA et al., 2019) pero es todavía escasa la bibliografía que aborda sus estudios desde los ámbitos de la educación no formal pese a que el uso de las tecnologías digitales y las redes sociales pueden resultar en oportunidades para la germinación de valores, actitudes y conocimientos que hagan de las experiencias de ocio digital referentes de calidad para la mejora de la vida y posibilidades de inclusión de los jóvenes menos afortunados (VASCOGONZÁLEZ; PÉREZ, 2017). Así, como señala Montes et al. (2019, p. 877) las redes sociales son un entorno en el que se fortalece el autoconcepto $y$, por ello, es necesario acercarnos a modelos educativos de ocio digital cuyo papel educativo vaya más allá del aula o centro educativo para integrarse dentro del espacio virtual compartido por lo jóvenes. Este espacio compartido a través de estrategias educativas sostenidas en TIC fomenta, como argumentan Campo et al. (2018, p. 622) la motivación hacia el aprendizaje y supone, entre otras ventajas, el fortalecimiento del vínculo entre los jóvenes y la mejora de los procesos de convivencia.

Hemos de observar por ello, que el valor pedagógico de estos espacios y herramientas tecnológicas adquieren relevancia cuando se usan como mediadores en contextos virtuales y facilitadores de procesos interactivos de construcción de conocimiento (SALMERÓN; RODRÍGUEZ; GUTIÉRREZ, 2010). De este modo la interacción participada por la tecnología va a permitir la consolidación de las relaciones sociales y, como exponen Sanmartín y Megías (2020 p. 53) puede ayudar a combatir procesos de soledad mediante la incorporación de los jóvenes a grupos virtuales en los que se comparten gustos y afinidades que permiten el empoderamiento de los jóvenes que participan en ellos y promueven el crecimiento mutuo y el aprendizaje colaborativo. Es esta perspectiva la que defienden Aguilar, Rubio y Viñals (2013, p. 199) cuando aseguran que debemos entender el ocio digital, especialmente el articulado en redes sociales, como un recurso de aprendizaje social "diferenciado de actividades de simple entretenimiento que facilita el conocimiento y beneficio personal y social". Entre esos beneficios asociados al ocio digital Farell (2012, p. 156) señala la posibilidad de trasmitir, entre otros, valores culturales, solidaridad, respeto o responsabilidad siempre que se haga un uso correcto y contextualizado de las mismas.

Tomando de referencia la exposición realizada hasta ahora, se plantea un trabajo cuya hipótesis se sostiene en la posibilidad de partir del interés que los jóvenes muestran en todo lo que hace referencia a la tecnología digital para articular, a través de ella, experiencias de ocio digital con valor educativo que permitan a los jóvenes en dificultad mejorar sus procesos de desarrollo personal y social. Con ello se pretende mejorar las competencias digitales de este colectivo social como medio para avanzar en sus posibilidades de inclusión en la sociedad de manera más justa y equilibrada. 


\section{Método}

La investigación propuso un paradigma cualitativo múltiple sostenido en el interés por contemplar un enfoque contextualizado que permita recoger de manera reflexiva el significado de la acción desde la perspectiva del propio sujeto (SCHENKEL; PÉREZ, 2018, p. 230).

\subsection{Objetivos}

Observando este propósito, el objetivo es investigar las experiencias de ocio digital de los jóvenes en dificultad social para descubrir las oportunidades educativas que se pueden implementar en el colectivo a través de actividades de ocio sostenidas en el entorno tecnológico. Para lograr este objetivo y dar respuesta a interrogantes de investigación como:

- ¿Es posible plantear espacios virtuales de ocio cuyo valor educativo mejore las posibilidades para el desarrollo personal y social de los jóvenes en dificultad social?

Se formularon un conjunto de objetivos específicos entre los que señalamos:

- Describir e interpretar el uso que hacen los jóvenes en dificultad social del ocio digital para identificar riesgos y oportunidades educativas que se derivan del uso de los mismos.

- Identificar alternativas de ocio digital con valor pedagógico para la mejora de las oportunidades de los jóvenes en dificultad social.

\subsection{Procedimiento y muestra}

Una vez determinados los objetivos de estudio, se planificó el acercamiento al campo elaborando un listado de las instituciones que respondían a los intereses de la investigación como trabajar en entornos vulnerables para la mejora de las posibilidades de inclusión social de los jóvenes de entre 16 y 21 . Atendiendo a estas características, se optó por uno de los programas de intervención socioeducativa que la administración pública de la Comunidad de Madrid (España) gestiona en un total de cinco centros (Tabla 1), distribuidos por todo el ámbito territorial de sus competencias, para garantizar una respuesta educativa accesible y organizada al total de jóvenes vulnerables de la capital.

Determinado el programa, la selección de participantes consideró como criterios básicos de clasificación: la paridad de género, asistencia regular a los programas socioeducativos, que fueran hispanohablantes, con adecuadas habilidades sociales y comunicativas, así como suficientes conocimientos tecnológicos. Bajo estos parámetros, contamos finalmente, con una muestra de 78 jóvenes cuyo contexto personal estaba sometido a procesos de dificultad social tales como escasez de recursos económicos, paternidad o maternidad precoz, falta de trabajo o empleo precario, inmigración y pequeña discapacidad. 
Belo Horizonte, v. 13, n. 3, p. 294-315, set.-dez. 2020 - ISSN 1983-3652 DOI : $10.35699 / 1983-3652.2020 .26132$

Tabla 1: Distribución de los centros por distritos Madrileños.

\begin{tabular}{cc}
\hline Código del centro & Distrito \\
\hline C1 & Centro, Retiro, Tetuán, Chamberí, Fuencarral El Pardo, Moncloa-Aravaca \\
\hline C2 & Salamanca, Chamartín, Moratalaz, Ciudad Lineal, Hortaleza, San Blas- \\
& Canillejas y Barajas \\
\hline C3 & Puente de Vallecas, Villaverde, Villa de Vallecas \\
\hline C4 & Arganzuela, Latina, Carabanchel y Usera \\
\hline C5 & Vicálvaro y Cañada Real \\
\hline
\end{tabular}

Fuente: Elaboración propia.

\subsection{Instrumento y proceso de análisis de datos}

Tras esta aproximación, se organizó el trabajo de campo eligiendo el método e instrumento más apropiado. Este debía estar al servicio del modelo de investigación escogido, paradigma cualitativo; las características de los informantes y el contexto en el que se situaba la investigación; adolescentes y dificultad social, así como al propósito de mejora de la realidad del colectivo. De modo que se concluyó con una fórmula de trabajo vertebrada sobre la investigación- acción que utilizaría como instrumento principal la entrevista abierta.

El diseño de la entrevista estuvo sometido a los criterios de validez de las investigaciones cualitativas definidos por los investigadores del National Research Council $^{2}$ Feuer, Towne, Shavelson $(2002$, p. 7) respetando dos propósitos esenciales: concentrar cuestiones relevantes para la investigación y mantener una relación directa con los objetivos programados, de forma que se redactaron, en torno a tres ámbitos: tecnología, ocio y educación, veintiocho preguntas que fueron sometidas a juicio de 10 expertos en la materia y supervisadas además, por la Subdirección General de Familia e Infancia del ayuntamiento de Madrid cuya valoración positiva sobre adecuación, contenido y claridad de las preguntas planteadas permitió verificar la validez del instrumento en su totalidad sin rectificar sus elementos.

Concluido este proceso, se accedió al programa socioeducativo en el que se efectuarían las entrevistas, durante los meses de julio y agosto de 2019, previa autorización de los participantes que fueron informados de los propósitos de la investigación, cuidando de no condicionar sus respuestas, así como de garantizar su anonimato cumpliendo con la ética propia de una investigación científica social (ANTOLÍNEZ; GARCÍA-CANO; BALLESTEROS, 2019, p. 131).

El proceso de análisis de la información tomó de referencia tres momentos fundamentales:

- Análisis de los datos recogidos antes de las sesiones. Permitió verificar la adecuación del trabajo al contexto.

- El análisis de los datos recogidos en las sesiones: posibilitando establecer las categorías iniciales o de primer nivel (Tabla 2).

- Análisis de la entrevista: mediante un profundo análisis de contenido acorde al modelo, método e instrumento de investigación elegido, auxiliada por el programa informático Atlas ti.

2 National Research Council (NRC): Consejo nacional de investigación de los EE. UU. Equivalente en España al Consejo Superior de Investigaciones Científicas. 
Tabla 2: Categorías de primer nivel.

\begin{tabular}{ccc}
\hline Categoría & Definición & Código \\
\hline Conexión & Gratuita, datos & G-H \\
\hline Tipo de usuario & Usuarios muy activos/ poco activos & UMA/UPA \\
\hline Actividad de Ocio & $\begin{array}{c}\text { Video juegos, aplicaciones móviles, } \\
\text { redes sociales }\end{array}$ & VJ/Apps/RRSS \\
\hline
\end{tabular}

Fuente: Elaboración propia.

El examen del nivel textual dio paso a la definición de las familias que articularía el segundo nivel de análisis o, conceptual, así se establecieron las relaciones entre los diferentes componentes del texto atendiendo a dos criterios de correspondencia, tema o significado, que utilizamos para definir las unidades de análisis que, sostenidas en las categorías centrales, permitieron la descripción y la interpretación de los datos (Tabla 3).

Tabla 3: Unidades de análisis y categorías centrales.

\begin{tabular}{ccl}
\hline \multicolumn{1}{c}{ Unidades de análisis } & \multicolumn{1}{c}{ Categorías centrales } \\
\hline Dificultad social y Experiencias digitales de Ocio & $\bullet$ & Prácticas de Ocio digital \\
& $\bullet$ & Redes sociales \\
\hline Riesgos del Ocio digital en dificultad social & $\bullet$ & Información falsa \\
& $\bullet$ & Peligros del ocio en red \\
\hline $\begin{array}{c}\text { Enfoque educativo para el ocio digital en la } \\
\text { juventud en dificultad social }\end{array}$ & $\bullet$ & Práctica Ocio digital \\
\hline
\end{tabular}

Fuente: Elaboración propia.

\section{Resultados}

El análisis de los datos reportó 98 códigos cuya recodificación, prescindiendo de los repetitivos o imprecisos, estableció un grupo final de 50 en el que comprobamos por el número de referencias encontradas en el discurso que algunos códigos sobresalían de los demás, evidenciando la correcta formulación de estos, así como su buen funcionamiento para el análisis (Figura 1).

Figura 1: Codificación y frecuencias.

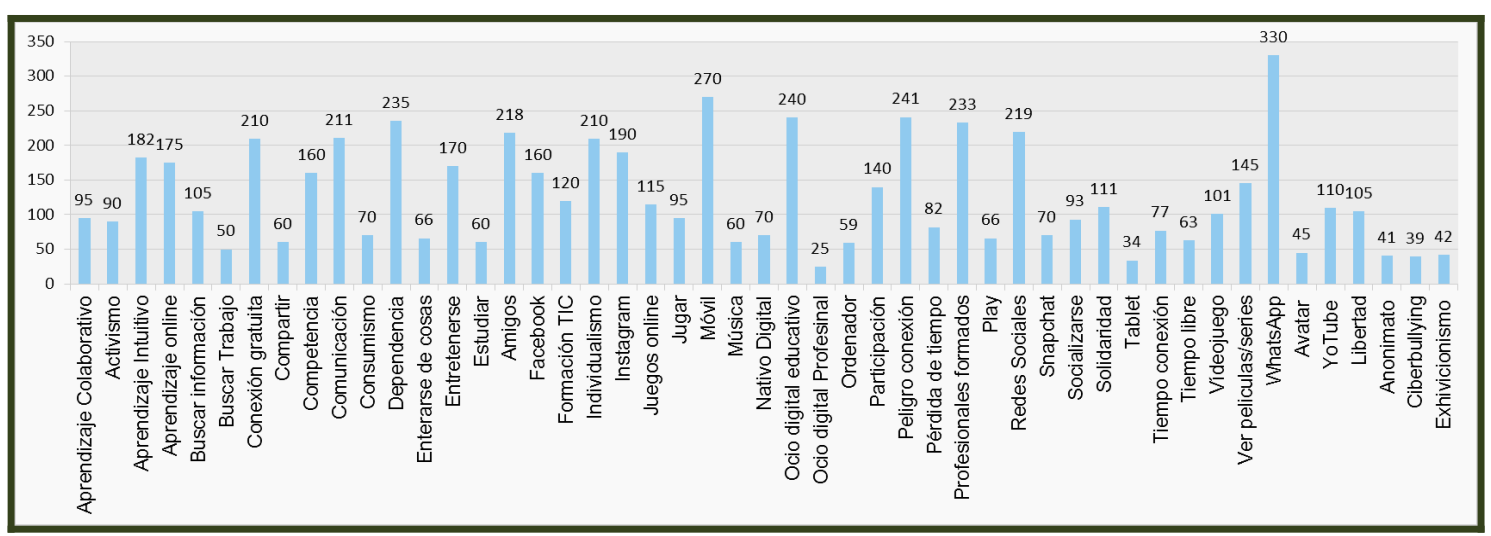

Fuente: Elaboración propia Atlas ti y Excel. 


\subsection{Procedimiento y muestra}

Las experiencias de ocio digital son, como evidencian los testimonios analizados, el conjunto de actividades que chicos y chicas en dificultad realizan por medio de la conexión Wifi a internet para las que utilizan, en exclusividad, su dispositivo móvil de última generación en detrimento de tablets u ordenadores considerados más adecuadnos para la realización de actividades educativas no de ocio. Respecto a ello afirman, que esta forma de conectarse gratuitamente, aun siendo conscientes del riesgo que supone, es el vehículo que usan a diario para el desarrollo de experiencias de ocio digital desde su hogar o en lugares públicos como centros comerciales y autobuses.

Al ser preguntados por la tipología de actividades reconocidas como parte de su ocio digital, responden similarmente en todas las entrevistas, destacándose en primer lugar el uso de las redes sociales a las que siguen YouTube y los videojuegos y juegos online.

Así mismo, coinciden las opiniones sobre la aplicación WhatsApp, a la que le otorgan un papel hegemónico para vivir experiencias de ocio a través de la red y con la que, como admiten, están en contacto continuo con los amigos del grupo para enterarse y comentar todo lo que ocurre dentro y fuera del entorno tecnológico. En este sentido, los jóvenes entrevistados, ante la cuestión del tiempo que pasan conectados a la red presentan, en la mayoría de sus respuestas, grandes dificultades para establecer un límite temporal definido, declarando con normalidad, que permanecen en conexión constante a su Smartphone combinando sus ocupaciones laborales, familiares o formativas con el uso de esta red social con la que, como evidencian, mantienen abierta una comunicación fácil y rápida en cualquier momento del día o la noche ya que, como han asegurado los entrevistados, llevan permanentemente su dispositivo móvil con ellos. Solamente siete informantes, declararon alejar de su alcance el teléfono o apagarlo durante alguna de sus horas lectivas (Figura 2).

Figura 2: Porcentaje de apagado del móvil durante las horas lectivas.

¿Cuándo apagas el móvil ?

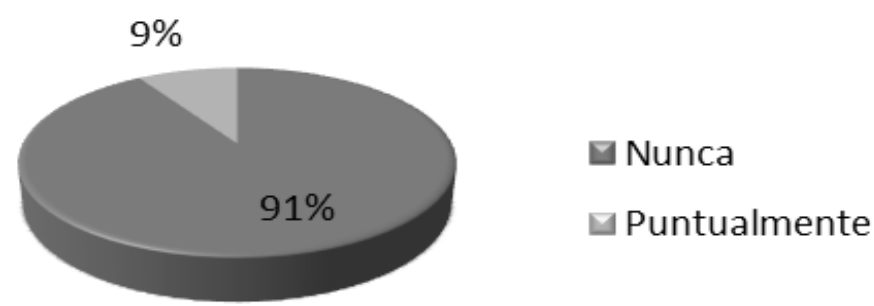

Fuente: Elaboración propia.

Otro de los argumentos coincidentes, cuando los jóvenes se refieren a las experiencias de ocio digital, es la posibilidad que les ofrecen como medio de distracción. Señalan que en momentos en los que están aburridos sin nada que hacer, utilizan el móvil para enviar WhatsApp, meterse en el perfil y fotos de sus contactos, buscar información de famosos, o buscar YouTubers de moda para copiar y compartir sus opiniones, comportamientos y estilo personal. Así lo evidencia la relación de asociación observada entre los códigos entretenerse, móvil y enterarse de cosas.

Cabe señalar, además, la relación que une estos códigos con la categoría ocio 
digital educativo que muestra también, que tanto chicos como chicas entrevistados son conscientes de que estar en conexión constante a la red, supone una pérdida de tiempo, que podría rentabilizarse en actividades con más valor educativo. No obstante, todos los testimonios reflejan, ante esta cuestión, que la posibilidad de perderse algo importante en la red, si no están conectados, es un hecho que les genera cierta ansiedad y desasosiego.

Por otro lado, para los jóvenes entrevistados, la interacción en la red está relacionada con la posibilidad para hacer y decir lo que quieran libremente pese a exponerse, como muestran los datos, de manera consciente a los riesgos que supone la falta de supervisión en la red, así se evidencia en la relación de causalidad entre los códigos dependencia y móvil. Significativamente se observa, además, respecto a la dependencia que los entrevistados reconocen que genera la red, la firme convicción del colectivo sobre la culpabilidad que tiene el entorno en el que han nacido. Afirman tajantemente que estar constantemente online es la consecuencia directa de haber crecido en una sociedad tecnologizada en la que existen numerosas empresas del sector digital que se apresuran a lanzar productos y crear aplicaciones con el propósito de mantenerles enganchados. Sobre esto, se observa nuevamente el vínculo que relaciona, el tiempo excesivo de conexión, con la falta de participación de los informantes en actividades de ocio con mayor valor para el desarrollo. La razón, como reflejan sus opiniones, es la insuficiente oferta de experiencias de ocio que se ponen al alcance de los jóvenes en dificultad social, ya sean en el entorno tecnológico o de la vida offline porque, como afirman el total de entrevistados, no pueden afrontar los gastos que suponen actividades más educativas como ir al cine, conciertos o, en concreto, se cita la feria del videojuego y la electrónica para el ocio, realizada anualmente en la capital Madrid Games Week "Semana del Juego de Madrid" (Figura 3).

Figura 3: Código con cita.

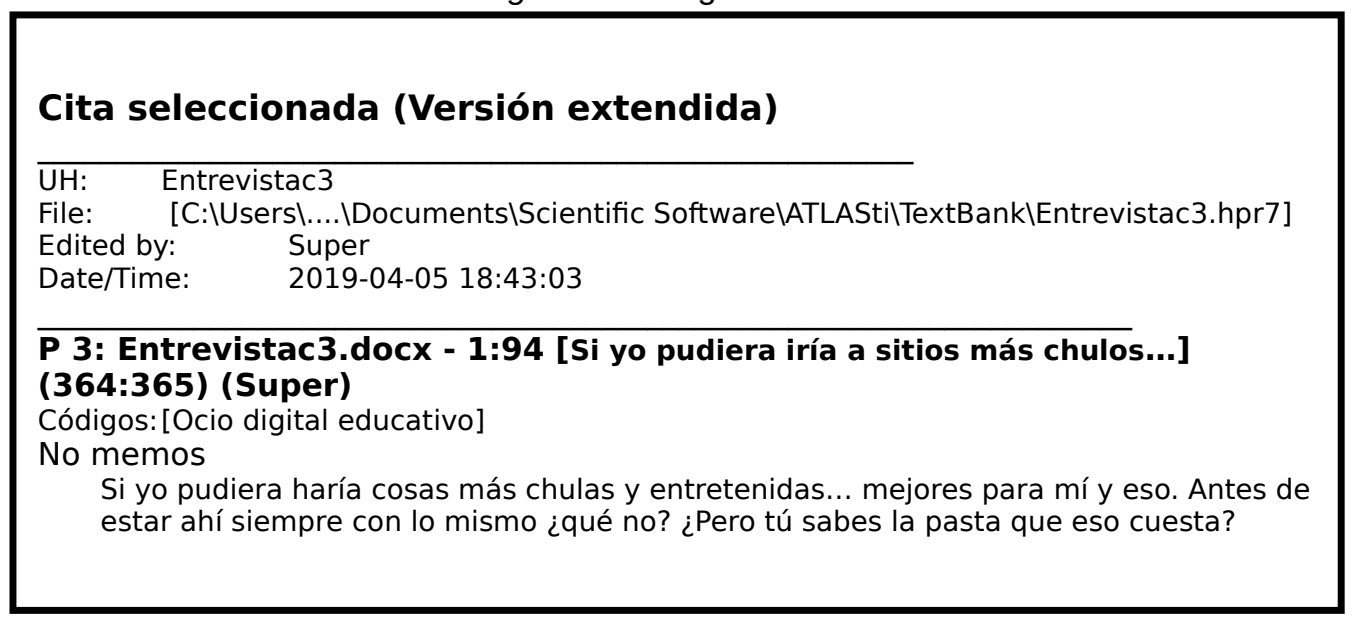

Fuente: análisis de la entrevista con Atlas Ti.

\subsection{Riesgos del ocio digital en dificultad social}

Continuando con el examen de la unidad hermenéutica abordamos el estudio referido a los riesgos que el ocio digital supone para los jóvenes más vulnerables.

En este sentido, entre las amenazas que los jóvenes identifican como más 
habituales dentro del ocio disfrutado en la red, aparece el uso de perfiles falsos. Desde esta perspectiva, el colectivo entrevistado advierte, que muchas de estas identidades son utilizadas para humillar o despreciar a personas, amparándose en el anonimato que les proporciona la red y la falta de control que en ella se ejerce. Las coincidencias entre las distintas entrevistas apuntan a que, este tipo de comportamiento no suele ser al azar, sino que los acosadores lo llevan a la práctica contra personas conocidas con las que tienen algún problema apareciendo, con frecuencia, alusiones a compañeros y profesores.

Joven C3: Codes: [Peligros de conexión] [Anonimato] [Ciberbullying]

Es que no tiene sentido...ósea que se hace porque lo sé, hay gente que le da igual y se mete con cualquiera, te insultan y eso. Pero lo normal es trolear ${ }^{3}$ a gente que sabes quién es, que te cae mal o algo de eso.

Se comprueba que los informantes, de los cinco centros visitados afirman, por un lado, saber de personas que llevan a la práctica este tipo de conductas y, por otro, tener en su entorno cercano algún conocido que padece $o$ ha padecido este tipo de comportamientos abusivos (Figura 4).

Figura 4: Acoso y redes sociales.

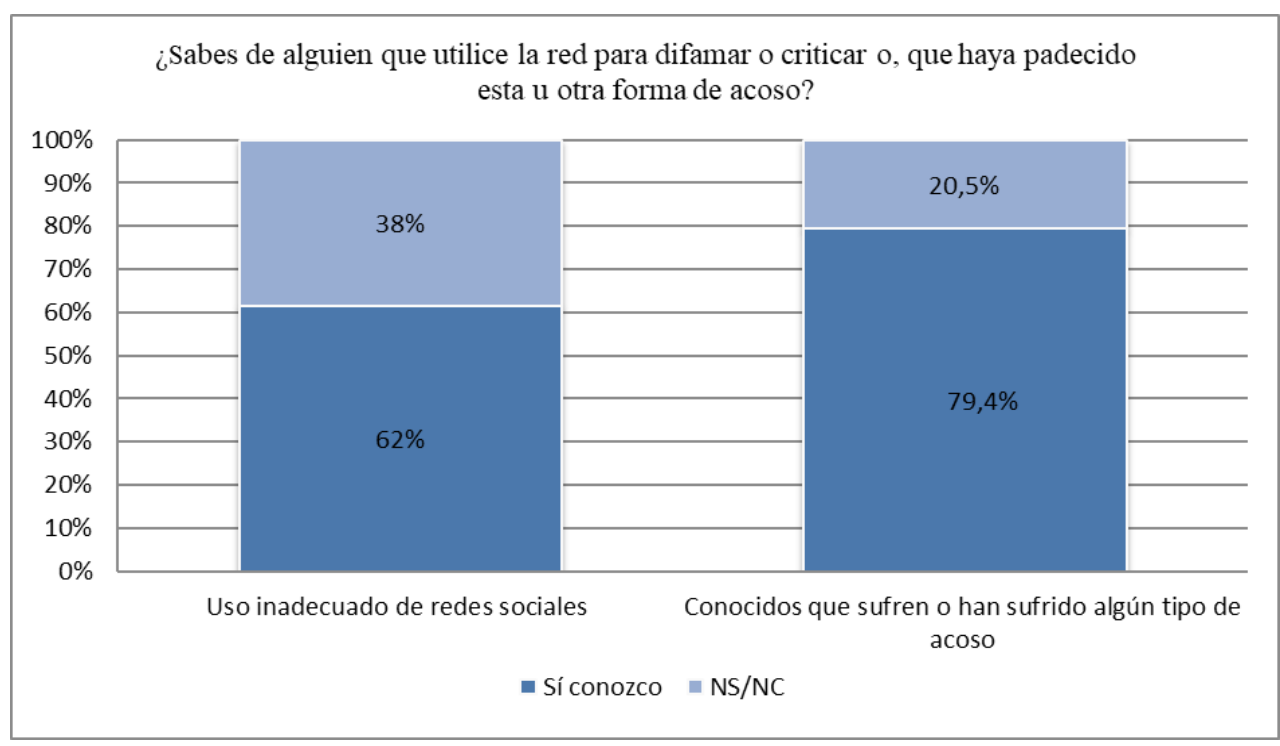

Fuente: análisis de las entrevistas.

La totalidad de los entrevistados reconoce su malestar ante estas actuaciones, pero los testimonios, ponen de relieve, su inacción ante estos comportamientos que, justifican en el temor a represalias, ausencia de consecuencias para los acosadores denunciados o falta de medidas contundentes de los centros y organismos públicos, a los que se acusa por parte del colectivo, de tratar el tema con indiferencia.

Cabe señalar otro punto de vista observado en muchas de las entrevistas, que justifican el uso de perfiles falsos como oportunidad para liberar las tensiones del entorno. Los informantes aseguran que participar con avatares les permite olvidar la realidad en la que viven $y$, crear un personaje divertido, hace que sientan que son admirados por otras

3 Trolear: Expresión utilizada en ámbito de la red que se utiliza para definir la intención de una persona o personas para hostigar a otro mediante la divulgación de mentiras o insultos. 
personas por medio de los comentarios formulados de manera interactiva. Además, los jóvenes sostienen que, esta forma de experiencia virtual permite sobreponerse a la timidez y participar de forma más activa y libre sin miedo a ser reconocidos. A su modo de ver, establecer relaciones en la red es más fácil que hacerlo de forma presencial y ello permite que no se sientan avergonzados gracias al anonimato proporcionado por su identidad falsa. En este sentido emerge del estudio de esta categoría, la tendencia de estos jóvenes a mantener relaciones a través del entorno digital con personas desconocidas.

Otra práctica de riesgo que se desprende, a la luz de los datos estudiados, es la que alude los navegadores como TorProjet ${ }^{4}$. A través de estas herramientas, disponibles de forma gratuita en la red, los jóvenes entrevistados reconocen que cualquiera tiene acceso a la compraventa de armamento, sustancias ilegales, pornografía, violencia, etc., sin riesgo de ser localizados. Sin embargo, pese a que la mayoría asegura conocer estos lugares virtuales, solo se evidencia un indicio claro de uso de este tipo de navegadores en las declaraciones recogidas en el centro 1.

Al margen de esta red, los datos resaltan que, tanto chicos y chicas, reciben mensajes o vídeos que tienen contenido incómodo o agresivo (peleas entre jóvenes, imágenes de pornografía o autoagresiones). Más de la mitad de los colaboradores, asegura, que les llegan de forma viral a través de amigos o personas de otros grupos en los que participan. Las opiniones coinciden en la dificultad para evitar ver y enviar esas imágenes cuando no son de violencia extrema o hacen comentarios de profesores $\mathrm{y}$, alegan en su defensa, que solamente las envían a su grupo de amigos.

Es significativa la falta de datos en las entrevistas que evidencien el conocimiento, dentro del colectivo, de las implicaciones legales sobre la tenencia y difusión de este tipo de contenidos resultando evidente, además, la escasa conciencia que los jóvenes tienen sobre la gravedad de este asunto al que restan importancia afirmando, que muchas de las imágenes que se envían por la red son falsas y, por ello las comparten para "echarse unas risas". No obstante, se aprecian diferencias significativas en varias citas que indican contrasentidos en este aspecto, ya que muestran un colectivo que afirma no saber distinguir claramente la información verdadera o falsa.

Categorías: [Enterarse de cosas] [Buscar información] [Formación TIC] Entrevistador: ¿Cómo distingues la información verdadera de la que no lo es? Joven C2: Pues no sé, la verdad que a veces es muy difícil... sobre todo porque es muy real todo lo que se manda y no te vas a poner a comprobarlo ¿no?

Joven C3: Hay cosas que se nota mogollón, pero es verdad que hay noticias... no sé de un famoso que, si ha hecho o dicho algo, que se ha ido con otra y tal...pues lo reenvío para cotillear un rato.

Joven C1: A mí me da igual, está en la red y me divierte, lo malo es cuando te cuelan una cosa y vas tú y la cuentas o lo mandas y los demás se parten... quedas como un pringao.

Igualmente representativos son los testimonios que coinciden en la actitud que manifiestan los progenitores ante las actividades que sus hijos implementan en casa mediante la conexión a internet. Así el análisis de contenido muestra un escaso o nulo interés de ambos padres sobre las experiencias que llevan a cabo los jóvenes de este colectivo que se evidencia, tal y como los jóvenes manifiestan, en la falta de control

4 TorProjet: Navegador que permite el acceso libre y anónimo a cualquier contenido que esté en la red mediante el triple del tráfico de los datos de un ordenador sin dejar rastro y permitiendo el acceso a sitios que la red local haya bloqueado. 
respecto a los tiempos y espacios de conexión e indiferencia sobre los contenidos que observan. Solo se han encontrado cinco testimonios que afirman haber sido reprendidos, en alguna ocasión, por estar conectados al móvil más allá de las doce de la noche.

Finalizamos el estudio del bloque señalando, otra de las amenazas que más vinculan los jóvenes con el ocio digital evidenciada en la relación de los códigos individualismo, dependencia, exhibicionismo y WhatsApp para los que se establecen relaciones de causalidad, correspondencia y asociación que indican la opinión de los jóvenes sobre el potencial que tienen estas experiencias, para generar dependencia a la conexión constante observada, por la mayoría de los informantes, como una actitud que provoca aislamiento del grupo de amigos y marginación social.

\subsection{Riesgos del ocio digital en dificultad social}

Los resultados de este bloque muestran la desconfianza que genera en el colectivo el concepto de ocio digital atendiendo a una perspectiva educativa. Más de la mitad afirma conocer páginas virtuales para compartir información sobre sus aficiones, interés o dudas, pero la mayoría evidencia la tendencia a no considerarlas para el ocio ya que, como afirman, presentan la información de una manera aburrida poco atractiva y monótona que no les resulta atractiva. No obstante, un $74 \%$ de los chicos y chicas entrevistados manifiesta, ante la posibilidad de utilizar redes $u$ otras aplicaciones, como herramienta para mejorar sus competencias, haberlo considerado siempre que les ofrezca un aprendizaje que tenga una aplicación útil y directa en su vida diaria. Atendiendo a esta idea, se destacan en el análisis, múltiples alusiones a llevar a la práctica este tipo de actividades en YouTube, a la que se relaciona con prácticas de Ocio digital como fuente de trasmisión de conocimiento a través de tutoriales de ayuda en pequeñas labores domésticas, resolución de dudas académicas o personales y vídeos para el entrenamiento personal. Por otro lado, los informantes, defienden con firmeza, las oportunidades que les brinda la aplicación WhatsApp para resolver dudas o preguntar de manera rápida, cuando en su grupo de amigos hay personas que tienen la información que ellos necesitan. La razón que esgrimen, tanto chicos y chicas, al ser preguntados ¿por qué? coincide en todos los centros en que esta forma de aprendizaje es mucho más sugerente y divertida, por ser, tal y como lo expresan, un medio más visual y cómodo que les permite retener información con más facilidad debido a que se presenta de forma más gráfica y con un lenguaje directo y claro.

Continuando con las prácticas relacionadas con el ocio digital, desde un enfoque educativo, se abren dos líneas en el análisis. Por un lado, las que observan el ámbito educativo de formación reglada y, por otro, el que hace referencia al plano no formal.

Comenzando por las segundas, los testimonios permiten observar el interés de los jóvenes ante esta posibilidad, ampliando la idea anterior, de que les ofrece una forma de aprendizaje más interactivo, cómodo y sencillo que se adapta a sus demandas y necesidades personales. Según el análisis de los testimonios, esta interacción les permite subir comentarios y fotos con información realizada por sus iguales o por ellos mismos, presentando el contenido que ellos quieren y que realmente les interesa. Como se desprende de sus comentarios, la oportunidad de crear de forma colaborativa cualquier información o contenido es el mejor de los aprendizajes y, por ello, consideran las redes sociales e internet, una herramienta que facilita la posibilidad de generar información, ideas y contenidos que, a través de prácticas digitales de forma lúdica, pueden convertirse 
en actuaciones con potencial educativo. Por otro lado, la categorización permite observar, ante la pregunta sobre cómo aprenden a utilizar estas redes sociales y aplicaciones, el mismo tipo de respuesta en todos los chicos y chicas entrevistados gracias a la coocurrencia de los códigos como: Aprendizaje intuitivo, Compartir, Aprendizaje colaborativo o Aprendizaje online.

Entrevistador: ¿Quién os enseña a manejar una App, red social?

Joven 1. C2. "Pues el otro (señala a su compañero) puedes mirar como lo hace".

Joven 2. C2. "Suele ser súper fácil".

Joven 1. C2. "O te enseña otra persona o lo investigas tú en la red".

Respecto al enfoque educativo formal, las entrevistas, indican que los informantes mantienen el interés manifestado en líneas anteriores. No obstante, se pone de relieve que el colectivo de jóvenes estudiado desconfía de la disposición de los profesores para llevar a la práctica una docencia que encaje con el aprendizaje en esta línea. La totalidad de entrevistados considera que los profesionales de la enseñanza desconocen las demandas que surgen del ámbito de la juventud en este escenario y, por ello, limitan su enseñanza digital a la difusión del temario a estudiar mediante vídeos en la red o, en explicaciones que muestran cómo funciona un sistema operativo. A este respecto, observamos mediante las categorías Profesionales formados; Ocio digital educativo; Aprendizaje intuitivo y Formación TIC vínculos que ponen de relieve, la necesidad de contar con personal educativo cuya formación, en Tecnologías de la Información y Comunicación, se ajuste a sus intereses y necesidades. Para los entrevistados, urge la puesta en marcha de actividades y propuestas educativas que fomenten experiencias de enseñanza-aprendizaje con estrategias que, sostenidas en soportes de tecnología digital, respondan a las demandas de los jóvenes para los que se programan.

Atendiendo a los testimonios examinados, los jóvenes se inclinan mayoritariamente por plantear lo que consideran una estrategia ideal de formación que debería poner en práctica un modelo educativo combinado, entre formación virtual y presencial porque señalan la importancia de mantener el contacto directo con los profesores y compañeros. En este sentido el análisis discursivo revela la naturalidad con la que los informantes admiten que, en una enseñanza íntegramente online, serían incapaces de mantener la atención directa sobre el contenido a trabajar. Así, coinciden los testimonios que aseguran la dificultad de estar trabajando en la red realizando una sola actividad y, confiesan su necesidad, de googlear ${ }^{5}$ o aprovechar, que nadie les controla, para estar al día de lo que ocurre en las redes sociales. Hecho que, tal y como lo observan los entrevistados, puede derivar en fracaso educativo.

Joven C5: Codes: [Aprendizaje online] [Pérdida de tiempo] [Enterarse de cosas] [Redes sociales] [WhatsApp] [Facebook].

¡A ver! Si es que tú imagina lo difícil que es estar ahí en la red...con las posibilidades que tienes para hacer lo que te dé la gana y, no, tienes que estar ahí pendiente de una sola y además que es de estudiar...pues tú, me parece chungo ¿no? Pues miras el WhatsApp o yo que sé. La verdad es que me parce difícil. A ver, que, si lo tienes que hacer lo haces, pero ya te va a costar más, seguro (Todos asienten).

Atendiendo a otra perspectiva ambos niveles educativos, formal y no prescriptivo deben abordar, acorde con la codificación realizada, la perspectiva del Ocio digital, desde 
una fórmula educativa que posibilite la colaboración e intervención directa de los jóvenes en el diseño y planificación de actuaciones dirigidas a la utilización de los tiempos y espacios de ocio digital.

En este sentido, emerge en las entrevistas estudiadas, el reclamo de los jóvenes, para hacerse con un entorno virtual en el que puedan ejercer de agentes educativos bajo la supervisión de un profesional del ocio digital cualificado. Un ejemplo de cómo llevarlo a la práctica, lo encontramos en la entrevista C3 que plantea, la formación de comunidades virtuales que implementen experiencias educativas de ocio digital arbitradas en torno a diferentes centros de interés, elegidos con los compañeros del grupo. En su opinión, el valor educativo de estas actividades supera las experiencias que se desarrollan en contextos educativos no digitales y, ponen de relieve, su entusiasmo por participar en actividades educativas, organizadas en las redes sociales, que les invitan a ser partícipes de grupos de trabajo, talleres o movilizaciones ciudadanas (Figura 5).

Figura 5: Enfoque educativo de Ocio digital.

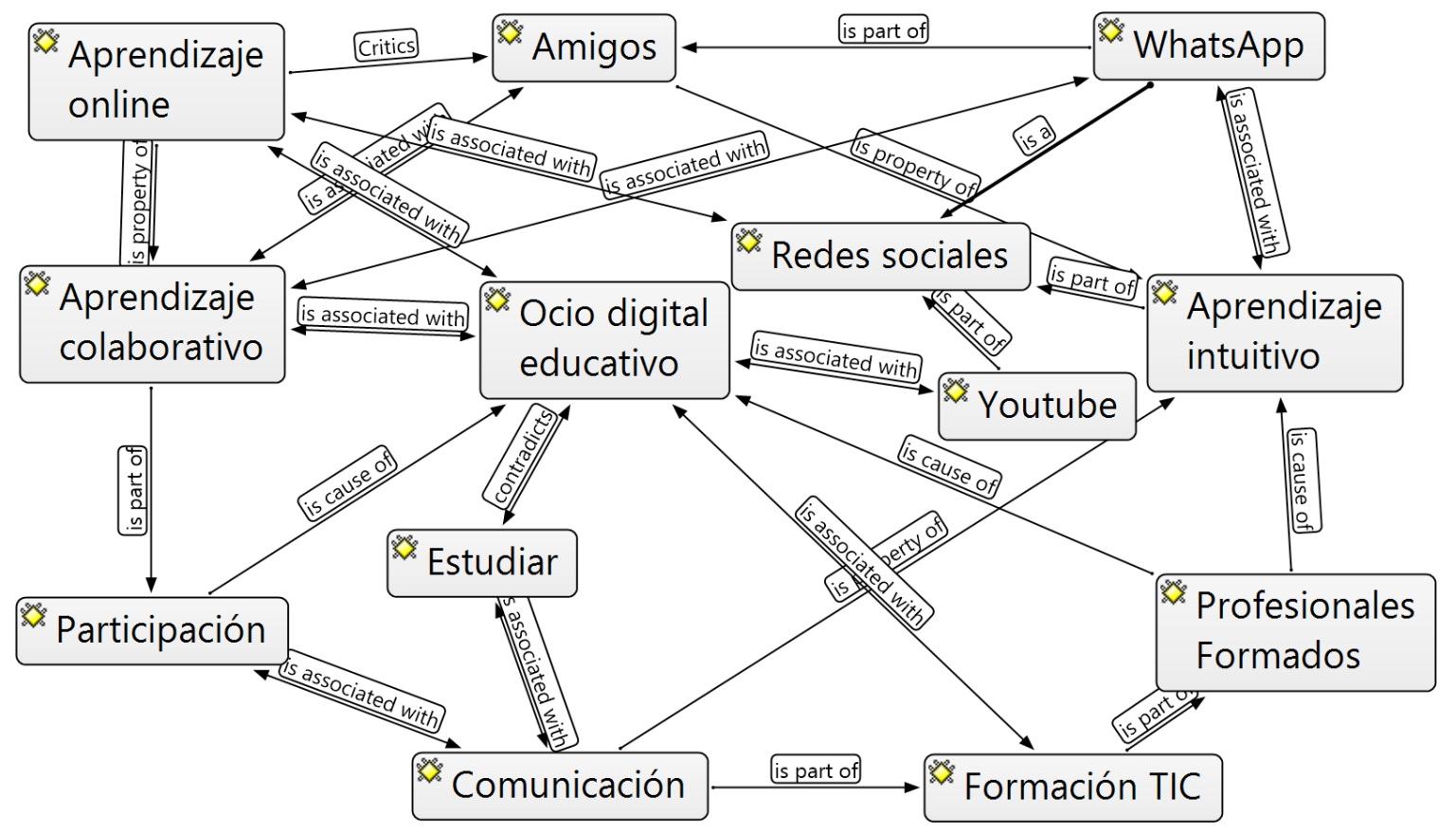

Fuente: Elaboración propia Atlas Ti.

Finalizamos el bloque, continuando con la visión de los jóvenes en dificultad, sobre lo que entienden debe ser educación en y a través de ocio digital, el análisis de la unidad hermenéutica devuelve constantes alusiones a la falta de conexión entre entorno digital del que son protagonistas los jóvenes y el contexto académico actual que califican como poco flexible y anticuado. Como muestran los datos, los informantes exteriorizan un fuerte rechazo a las actividades teóricas, otorgándole gran importancia, a las actividades digitales que ofrecen un enfoque formativo, cuya aplicación directa en su vida diaria, les resulte útil para acometer su proceso de independencia.

En el discurso, chicos y chicas han destacado, como ejemplo, el protagonismo de la tecnología en el sector productivo, manifestando su malestar, sobre las escasas posibilidades para acceder a la formación que, en tecnología digital, demanda el mercado 
de trabajo. Tal y como coinciden en afirmar escasean los planes educativos en formación obligatoria, aclaran, que cuenten con contenidos de este tipo $\mathrm{y}$, si los hay, son poco sugerentes y se trabajan desde un plano teórico que no se parece a las prácticas de ocio digital educativo que ellos conciben.

\section{Discusión y conclusiones}

Las actividades de ocio digital que llevan a cabo los jóvenes en dificultad social pueden describirse comenzando con las afirmaciones del estudio de Melendro, GarcíaCastilla y Goig (2016) que aseguran que la conexión Wifi y el smartphone son los elementos que este colectivo utiliza a diario para disfrutar de sus espacios de ocio dentro del entorno digital. En este medio virtual, para el que no se determinan límites temporales, descubrimos un colectivo de jóvenes que concibe el ocio digital como un espacio de conexión continua a lo largo del día en el que alternan otras actividades de índole personal o académico. Esto es posible coincidiendo con las afirmaciones de Pastor, Martín y Montes (2019, p. 1006) porque estos jóvenes permanecen junto a su smartphone las veinticuatro horas del día en una experiencia de comunicación compartida con los amigos facilitada por la conexión continuada a su aplicación favorita, WhatsApp. El principal objetivo de esta actividad es mantenerse informados, de forma incesante, de todo lo que ocurre dentro y fuera de la red por miedo a perderse algo importante si desconectan el móvil. Es evidente que esta forma de participar en la red confirma una de las cuestiones más controvertidas en los estudios actuales sobre juventud y tecnología que relaciona, el uso de los dispositivos móviles e internet, con la dependencia (CABERO et al., 2020) y que repercute, coincidiendo con el trabajo de Hernández et al. (2019, p. 43) en el aislamiento personal y social de los jóvenes.

Una dependencia que, dentro de este colectivo, se descubre asociada a la sociedad tecnológica, ante la que los jóvenes en dificultad social no manifiestan capacidades suficientes para evitar el consumo de aplicaciones digitales de valor educativo cuestionable $\mathrm{y}$, vinculada a su escaso poder adquisitivo, que limita sus oportunidades para disfrutar de actividades de ocio digital con más valor educativo, ya que suponen un desembolso económico que en su situación de dificultad es inasumible.

Otro de los usos que se pueden describir entre las experiencias de ocio digital de los jóvenes en dificultad social, además del comunicativo, es el que responde como exponen Montes et al. (2019, p. 872) a su necesidad de entretenimiento o recreación para lo que recurren, como se ha verificado, a actividades con escasa repercusión educativa ya que se centran, principalmente, en la recepción de información a través de las redes sociales o YouTube para repetir los patrones de comportamiento y opinión de personajes, a los que idealizan y dan credibilidad, que se conocen como influencers.

Cabe señalar al respecto, que se revela un colectivo juvenil poco crítico con la información que recibe a través de las redes, hecho que permite observar por un lado, la diferencia con los resultados del estudio de Pastor, Martín y Montes (2019, p. 1005) sobre el acceso a la información a través de internet que tienen los jóvenes madrileños, en el que los datos indicaban lo contrario y, por otro, que los jóvenes en dificultad social, como señalaba Vasco-González (2018) presentan menores competencias para procesar y distinguir la información que reciben de las redes sociales y que simplemente se entretienen con la difusión de contenidos para los que no han comprobado su veracidad 
(SANMARTÍN; MEGÍAS, 2020).

A esta falta de sentido crítico se suma, que los jóvenes en dificultad social son conscientes del riesgo al que se exponen mediante las prácticas de ocio digital, pero presentan una tendencia a normalizar, minimizar o no dar valor a sus consecuencias que se justifica como sostienen Montes-Vozmediano, García-Jiménez y Menor-Sendra (2018, p. 68) porque forma parte del proceso de reafirmación de la adolescencia.

Esto explicaría que los jóvenes del colectivo observen con normalidad que el uso de perfiles falsos, para la realización de actividades tan controvertidas como el ciberacoso, sea una de las experiencias más habituales en las redes digitales. Si bien queda acreditado su malestar ante estas actividades, también se percibe la pasividad y falta de autocrítica, frente a unas prácticas de las que desconocen sus implicaciones legales y que consideran impunes por la ausencia de compromiso y actuaciones contundentes por parte de los centros educativos y organismos públicos. En este sentido, se hace necesaria la crítica sobre un comportamiento del que los jóvenes en dificultad social no asumen responsabilidades, pese a que se ha evidenciado su participación en grupos de WhatsApp, en los que se difunden contenidos ofensivos y violentos, que pueden resultar nocivos en el desarrollo personal y social propio así como en el de sus iguales. Se ha comprobado además, que dentro de este colectivo social hay jóvenes que conocen fórmulas de acceso a navegadores difíciles de rastrear que permiten adentrarse, de manera oculta, en webs que facilitan actividades tan arriesgadas como la compra de armas o estupefacientes. Esta conducta, evidentemente peligrosa, se ve reforzada por un entorno familiar que apenas ejerce la mediación necesaria que recomiendan Gordo et al. (2019, p. 30) en el control o restricciones de los contenidos que los jóvenes visualizan en las redes.

Parece evidente que el principal problema que deben resolver los jóvenes en dificultad social para hacer frente a los peligros que se derivan de sus prácticas de ocio en el entorno digital, se encuentra en la mejora de sus competencias tecnológicas, principalmente respecto a la información y contenidos que ellos observan. En este sentido, el trabajo evidencia el interés que estos jóvenes muestran ante la posibilidad de mejorar sus competencias, a través de aprendizajes sostenidos en prácticas de ocio digital, circunstancia que además de corroborar la hipótesis de la que partíamos al inicio de esta investigación, nos permite cumplir con el objetivo planteado de encontrar oportunidades educativas.

Así se descubre que los jóvenes en dificultad social no confían en la formación obligatoria como vehículo para el desarrollo de experiencias de ocio digital de corte educativo porque consideran, que el modelo de educación actual tiene un enfoque, respecto al ámbito de la tecnología, que no responde a las demandas digitales reales de los jóvenes en la sociedad actual González y Gutiérrez (2017). Esta desconfianza se observa además, en sus propias posibilidades de asumir el compromiso que conlleva trabajar, en una sola actividad en la red, sin articular otras prácticas de ocio digital que les distraigan de su verdadero propósito. Por ello, se hace necesario en el colectivo, la figura de una persona de referencia que conduzca su aprendizaje a través de un trato directo y una educación personalizada que actúe como mediador en las experiencias de ocio digital para que éstas faciliten, de acuerdo con Salmerón, Rodríguez y Gutiérrez (2010, p. 165) el alcance de los conocimientos y competencias necesarias para mejorar su situación particular y sus posibilidades de avanzar en el proceso de inclusión independencia.

Esta realidad refleja la posibilidad de plantear una estrategia educativa contextualizada de manera flexible en ambos escenarios, virtual y presencial y ámbitos 
educativos formal y no formal, para implementar lo que los jóvenes en dificultad social consideran un paradigma ideal para el desarrollo de valores y competencias de aprendizaje a través del ocio digital.

Este modelo debe considerar experiencias de ocio digital que promuevan la relación con los iguales por medio de la formación de comunidades virtuales, en las que los jóvenes en dificultad puedan compartir los temas de interés que previamente han consensuado con los compañeros. De esta forma, además de la promoción del aprendizaje colaborativo y social, estas actuaciones pueden ayudar a combatir los procesos de aislamiento que sufren algunos de estos jóvenes así como a superar su timidez por medio de la participación en el escenario digital (AGUILAR; RUBIO; VIÑALS, 2013; SANMARTÍN; MEGÍAS, 2020). Cabe señalar al respecto que se comprueba, en este modelo educativo de ocio, la promoción de los beneficios que Farell (2012) asociaba al ocio digital como el respeto o la solidaridad cuando estas experiencias se desarrollaban en contextos adecuadamente contextualizados.

Otro hallazgo importante sobre las alternativas educativas que se pueden impulsar en esta línea es, el que tiene por objetivo, que los jóvenes en dificultad sean participantes activos en la construcción de su proceso educativo en la red, así, las facultades que han manifestado para el aprendizaje intuitivo y colaborativo posibilitan la creación y difusión de contenidos digitales que se ajustan a sus necesidades formativas e intereses reales. Intereses que, para los jóvenes en circunstancias difíciles, se dirigen principalmente al perfeccionamiento de competencias digitales que mejoren sus aptitudes educativas y posibilidades de acceso al mercado de trabajo y, con ello, sus expectativas de independencia e inclusión en la sociedad.

Atendiendo a esta exposición finalizamos señalando la importancia de seguir investigando en este contexto ya que, en la actualidad, son escasos los estudios en referencia al ámbito de la juventud en dificultad social y el ocio digital. Así, como se ha puesto de manifiesto en esta investigación, la intervención educativa dentro de este grupo tiene en la tecnología un amplio campo de estudio con el que poder ayudar, desde perspectivas diferenciadas, a cumplir con el objetivo de mejora de la realidad de este colectivo social.

\section{Referencias}

AGUILAR, G. E.; RUBIO, F. I. Y.; VIÑALS, B. A. El ocio digital como recurso para el aprendizaje, la socialización y la generación de capital social. Revista de Sociología de la Educación-RASE, v. 6, n. 2, p. 196-209, 2013.

ALARCÓN, S. M. La Sociedad Del (Des)Conocimiento en la era informacional. En: LINDÍN, C.; RIVERA-VARGAS, P.; PITARQUE, M. (eds.). Tecnologías digitales para transformar la sociedad. Universitat de Barcelona: LiberLibro, v. 2, 2019. p. 174-184.

ANTOLÍNEZ, D. I.; GARCÍA-CANO, M.; BALLESTEROS V. B. Aproximación al método etnográfico. In: BALLESTEROS, B. (Coord.). Investigación Social Desde La Práctica Educativa. Madrid. UNED, 2019. p. 103-159.

AREA, M.; GUARRO, A. La alfabetización informacional y digital: fundamentos 
pedagógicos para la enseñanza y el aprendizaje competente. Revista española de documentación científica, n. 35, p. 46-74, 2012.

AVILÉS, P. Que no nos engañen: decálogo para hacernos cazadores de "fake news", 2018, Disponible en: https://url2.cl/pWh4e. Acceso em: 28 apr. 2020.

BALLESTEROS, J. C.; PICAZO, S. L. Las TIC y su influencia en la socialización de los jóvenes. Centro Reina Sofía sobre Adolescencia y Juventud, Fundación de ayuda contra la drogadicción (FAD), 2018.

BERTONE, R.; FILIPPI, J. L.; LAFUENTE, G. J.; BALLESTEROS, C. A.; LAFUENTE, G.; PÉREZ, D.; MANSILLA, G. A. Aprendizaje mediado por tecnología móvil. In: XXI Workshop de Investigadores en Ciencias de la Computación (WICC 2019, Universidad Nacional de San Juan), 2019. Disponible en: http://sedici.unlp.edu.ar/bitstream/handle/10915/77324/Documento_completo.pdfPDFA.pdf?sequence=1\&isAllowed=y. Acceso em: 28 apr. 2020.

CABERO A. J.; MARTÍNEZ, P. S.; VALENCIA O, R.; LEIVA, J. P.; ORELLANA, M. L.; Y HARVEY L. I. C. La adicción de los estudiantes a las redes sociales on-line: un estudio en el contexto latinoamericano. Revista complutense de Educación, v. 31, n. 1, p. 1-11, 2020.

CALVO, S. T.; Y LUQUE, S. G. Adultos y jóvenes ante las fakenews ¿quién comparte más mentiras? In: Redes sociales, tecnologías digitales y narrativas interactivas en la sociedad de la información. Interamericana de España: McGraw-Hill, 2019. p. 43-54.

CAMPO, A.; SIERRA, A.; BARRIOS, B.; CABALLERO, C.; BERMUDEZ, C.; OROZCO, C.; BARRIOS, D.; CONTRERAS, D.; ARAGON, E.; OROZCO, E.; CONTRERA, F.; MEDINA, F.; PEREA, G.; ACUÑA, G.; OCHOA, H.; DIAZ, H.; ALEMAN, J.; CARVAJAL, J.; BARRIOS, J.; “...” Y ANAYA, U. Práctica de valores apoyada en las tecnologías de la información y la comunicación como estrategia para la sana convivencia. Cultura. Educación y Sociedad, v. 9, n. 3, p. 619-626. 2018. DOI: http://dx.doi.org/10.17981/cultedusoc.9.3.2018.73

CARO, S. Información y verdad en el uso de las redes sociales por parte de adolescentes. Teoría de la educación. Revista interuniversitaria, v. 27, n. 1, p. 187-199, 2015.

CUBEROS, R. C.; SÁNCHEZ, M. C.; CAMPOS, G. G.; ORTEGA, F. Z. Victimización en la escuela, ocio digital e irritabilidad: análisis mediante ecuaciones estructurales. Relieve: Revista ELectrónica de Investigación y EValuación Educativa, v. 24, n, 1, p. 1-12, 2018.

CUENCA, M. Ocio Valioso. Documentos de Estudio de Ocio, 52. Bilbao. Universidad de Deusto. 2014.

DE VALENZUELA BANDíN, Á. L.; GRADAÍlLE PERNAS, R.; CARIDE GÓMEZ, J. A. Las prácticas de ocio y su educación en los procesos de inclusión social: un estudio comparado con jóvenes (ex) tutelados en Cataluña, Galicia y Madrid. Pedagogía social: revista interuniversitaria, n. 31, p. 33-47, 2018. 
FARELL, V. G. E. Formación de valores mediante las tecnologías de la información y las comunicaciones. Revista Cubana de Informática Médica, v. 4 n. 2, p. 154-160, 2012.

FEUER, M. J.; TOWNE, L.; SHAVELSON, R. J. Scientific Culture and Educational Research. Educational Researcher, v. 8, n. 31 p. 4-14. 2002. DOI: 10.3102/0013189X031008004

GONZÁLEZ, F. Jóvenes y participación. Estudio sobre participación juvenil. Vitoria. Consejo de la Juventud de Euskadi. 2013. Disponible em: https://n9.cl/h65n. Acceso 30. abr. 2020.

GONZÁLEZ, G. A.; CAMPOY, T. P. Ciberacoso y cyberbullying: diferenciación en función de los precipitadores situacionales. Revista Española de Investigación Criminológica, n. 16, p. 1-31, 2018.

GONZÁLEZ, R. R.; GUTIÉRREZ, M. A. Competencias mediática y digital del profesorado e integración curricular de las tecnologías digitales. Revista Fuentes, v. 19 n. 2, p. 57-67, 2017.

GORDO, L. A.; DE RIVERA, J.; DÍAZ, C. C.; GARCÍA, A. A Factores de socialización juvenil estudio Delphi. Madrid: Centro Reina Sofía sobre adolescencia y juventud y Fundación de ayuda contra la drogadicción (FAD), 2019.

GRANADO, P. M. Educación y exclusión digital: Los falsos nativos digitales. Revista de Estudios Socioeducativos. ReSed, n. 7, p. 27-41, 2019.

HERNÁNDEZ, C. J.; ORTIZ, M. M.; MARTÍNEZ A. J.; RAMÍREZ, A.; MIRAMONTES, A. Adicción a Internet. Enseñanza e Investigación en Psicología, n. especial, p. 34-45, 2019.

MAROÑAS BERMÚDEZ, A.; MARTÍNEZ GARCÍA, R.; GRADAÍlLE PERNAS, R. Educación del ocio en y con la comunidad. Aportes desde la pedagogía social. Perfiles educativos, v. 41, n. 163, p. 111-126, 2019.

MARÍN-DIAZ, V.; CABERO-ALMENARA, J. Las redes sociales en educación: ¿desde la innovación a la investigación educativa? RIED. Revista Iberoamericana de Educación a Distancia, v. 22, n. 2, p. 25-33. 2019. DOI: http://dx.doi.org/10.5944/ried.22.2.24248

MELENDRO, M.; GARCÍA-CASTILLA, F. J.; GOIG, R. Influencia del uso de las TIC en las expectativas de los jóvenes vulnerables. Revista española de Pedagogía, v. 7, n. 263, p. 71-89. 2016.

MONTES-VOZMEDIANO, M.; GARCÍA-JIMÉNEZ, A.; MENOR-SENDRA, J. Los vídeos de los adolescentes en YouTube: características y vulnerabilidades digitales. Comunicar: Revista científica iberoamericana de comunicación y educación, n. 54, p. 61-69, 2018. DOI: https://doi.org/10.3916/C54-2018-06

MONTES, C. M.; GRACIA, O. P.; VALENCIA, C. F.; OLIVEROS, R. L. Modelo interdisciplinar para el estudio de jóvenes y autoconcepto a partir del consumo de redes 
sociales virtuales. In: ROIG-VILA (ed.) Investigación e innovación en la enseñanza superior. Nuevos Contextos, nuevas ideas. Barcelona: Octaedro 2019, p. 871-879.

PASTOR, R, Y.; MARTÍN, N, R.; MONTES, V. M. Patrones de uso, control parental y acceso a la información de los adolescentes en la red. Estudios Sobre El Mensaje Periodístico, v. 25, n. 2, p. 995-1012, 2019.

PEÑA, H, M. Á.; RUEDA, L, E.; PEGALAJAR, P. M. ${ }^{a}$ C. Posibilidades didácticas de las redes sociales en el desarrollo de competencias de educación superior: percepciones del alumnado. Píxel-Bit. Revista de Medios y Educación, n. 53, p. 239-252, 2018.

PONCE-DE-LEÓN-ELIZONDO, A.; SANZ-ARAZURI, E.; SÁENZ-DE-JUBERA-OCÓN, M.; ALONSO-RUIZ, R. A. La jornada lectiva a debate para (re) convertir los centros educativos en espacios para el ocio. Revista de Investigación Educativa, v. 37, n. 2, p. 395-411, 2019.

PRATS, F. M. Á.; RODRÍGUEZ, T. A.; OBERST, U.; CARBONELL, X. Diseño y aplicación de talleres educativos para el uso saludable de internet y redes sociales en la adolescencia: descripción de un estudio piloto. Pixel-Bit, n. 52, p. 111-124, 2018.

RIVADULLA LÓPEZ, J. C.; RODRÍGUEZ CORREA, M. Ciberacoso escolar: experiencias y propuestas de jóvenes universitarios. RIED. Revista Iberoamericana de Educación a Distancia, v. 22, n. 2, p. 179-201, 2019. DOI: http://dx.doi.org/10.5944/ried.22.2.23541

RODRÍGUEZ, E.; BALLESTEROS, J. C. Jóvenes, ocio y TIC. Una mirada a la estructura vital de la juventud desde los referentes del tiempo libre y las tecnologías. Madrid: Centro Reina Sofía sobre Adolescencia y Juventud, FAD. 2019. DOI: 10.5281/zenodo.3537638

SALMERÓN, H.; RODRÍGUEZ, S.; GUTIÉRREZ, C. Metodologías que optimizan la comunicación en entornos de aprendizaje virtual. Comunicar, v. 34, n. 17, p. 163-171, 2010.

SÁNCHEZ PADILLA, M. L.; LAZCANO ORTIZ, M.; ARIAS RICO, J.; JIMÉNEZ SÁNCHEZ, R. Tecnologías de la Información y la Comunicación (TIC) para fortalecer el aprendizaje significativo de los estudiantes. Educación y Salud Boletín Científico Instituto de Ciencias de la Salud Universidad Autónoma del Estado de Hidalgo, v. 8, n. 15, p. 116-118, 2019 DOI: https://doi.org/10.29057/icsa.v8i15.4925

SANMARTÍN, A.; MEGÍAS, I. Jóvenes, futuro y expectativa tecnológica Madrid: Centro Reina Sofía sobre Adolescencia y Juventud, 2020. DOI: 10.5281/zenodo.3629108

SCHENKEL, E., PÉREZ, M. I. Un abordaje teórico de la investigación cualitativa como enfoque metodológico. Acta Geográfica, v. 12, n. 30, p. 227-233, 2018.

TUMINO, M. C.; BOURNISSEN, J. M. Una mirada a la integración de los valores desde el rol de las TIC. Ciencia, docencia y tecnología, v. 28 n. 54, p. 188-215, 2017.

VASCO-GONZÁLEZ, M. Ocio digital, sociedad de la Información y Jóvenes en dificultad 
social: Experiencias educativas en contextos virtuales, 2018, 443, Tesis doctoral Facultad de educación, Universidad Nacional de Educación a distancia, Madrid, 2018.

VASCO-GONZÁlEZ M.; PÉREZ, S, G. Ocio digital en los jóvenes en dificultad social. Bordón. Revista de pedagogía, v. 69, n. 2, p. 147-160, 2017.

VASCO-GONZÁlEZ, M.; GOIG, M. R.; ÁLVAREZ, F. A. Elementos contextuales que dificultan la inclusión sociodigital de los jóvenes vulnerables. En: AZNAR, D, I.; CÁCERES, R. M. ${ }^{a}$. P.; ROMERO, R. J. M.; MARTÍN, M. J. (eds.). Investigación e innovación educativa. Madrid: Dykinson, 2020. p. 151-164.

VÁZQUEZ, B., T. De las audiencias televisivas a las redes sociales. Convergencia de medios en la sociedad digital. Metamorfosis. Revista del centro Reina Sofía sobre adolescencia y juventud, n. 10, p. 77-89, 2019.

Recebido em dia 12 de maio de 2020. Aprovado em dia 04 de agosto de 2020. 